\title{
6-color/1-target immuno-SERS microscopy \\ on the same single cancer cell \\ SUPPORTING INFORMATION
}

Elzbieta Stepula1, Xin-Ping Wang1, Supriya Srivastav', Matthias König ${ }^{1}$, Janina

Levermann $^{2}$, Sabine Kasimir-Bauer ${ }^{2}$ and Sebastian Schlücker ${ }^{1 *}$

* Sebastian Schlücker E-mail: sebastian.schluecker@uni-due.de

${ }^{1}$ Department of Chemistry and Center for Nanointegration Duisburg-Essen

(CENIDE), University of Duisburg-Essen, Universitätsstr. 5, Essen 45141, Germany

2Department of Gynecology and Obstetrics, University Hospital Essen, University of

Duisburg-Essen, Hufelandstr. 55, D-45122 Essen, Germany 


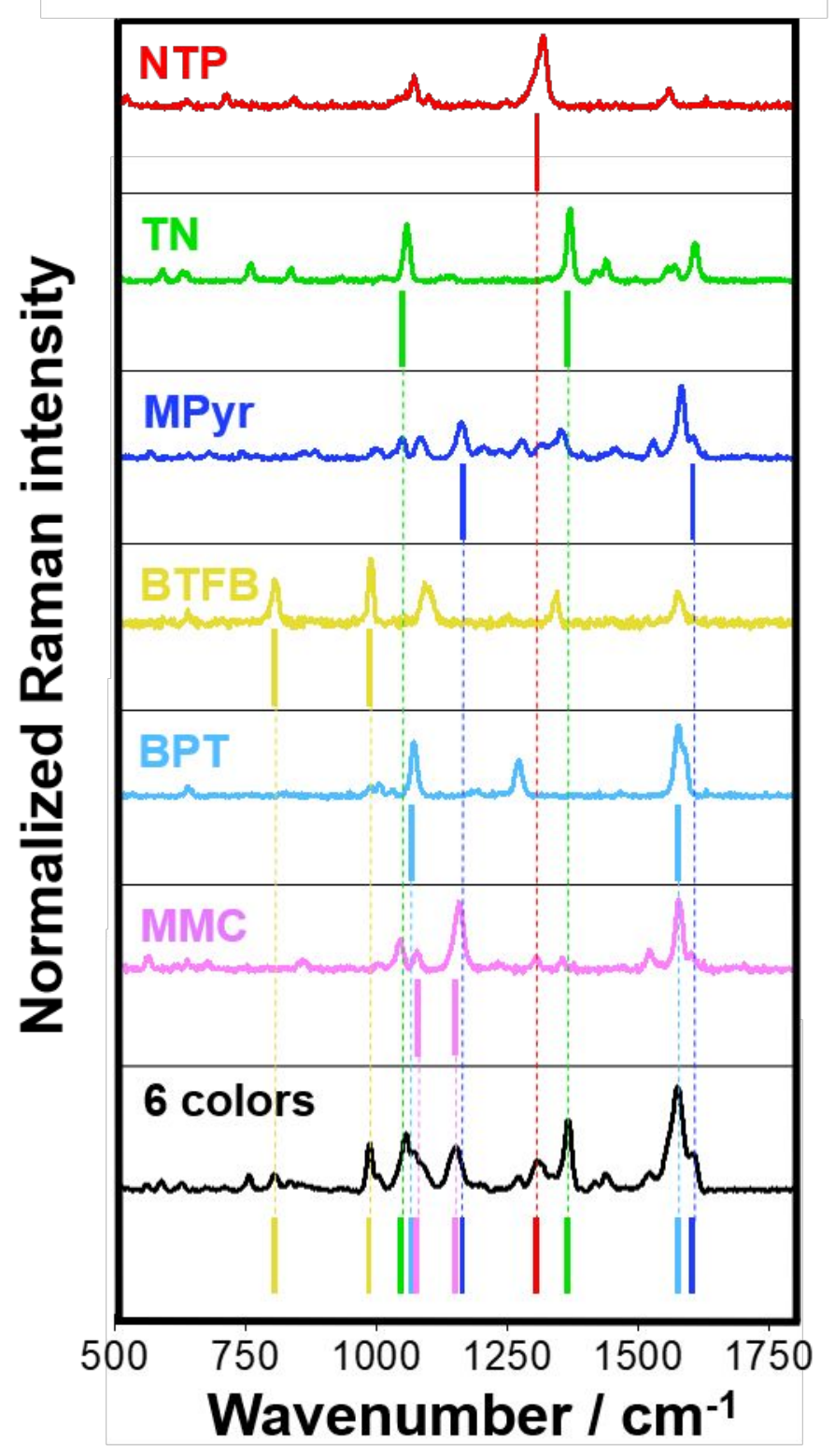

Figure S1. SERS spectra of six SERS nanotags functionalized with six different Raman reporter molecules (NTP - red, TN - green, MPyr - blue, BTFB - yellow, BPT - cyan, MMC - magenta) and the mixture of all six (black). For each spectrum corresponding bar code is shown. 

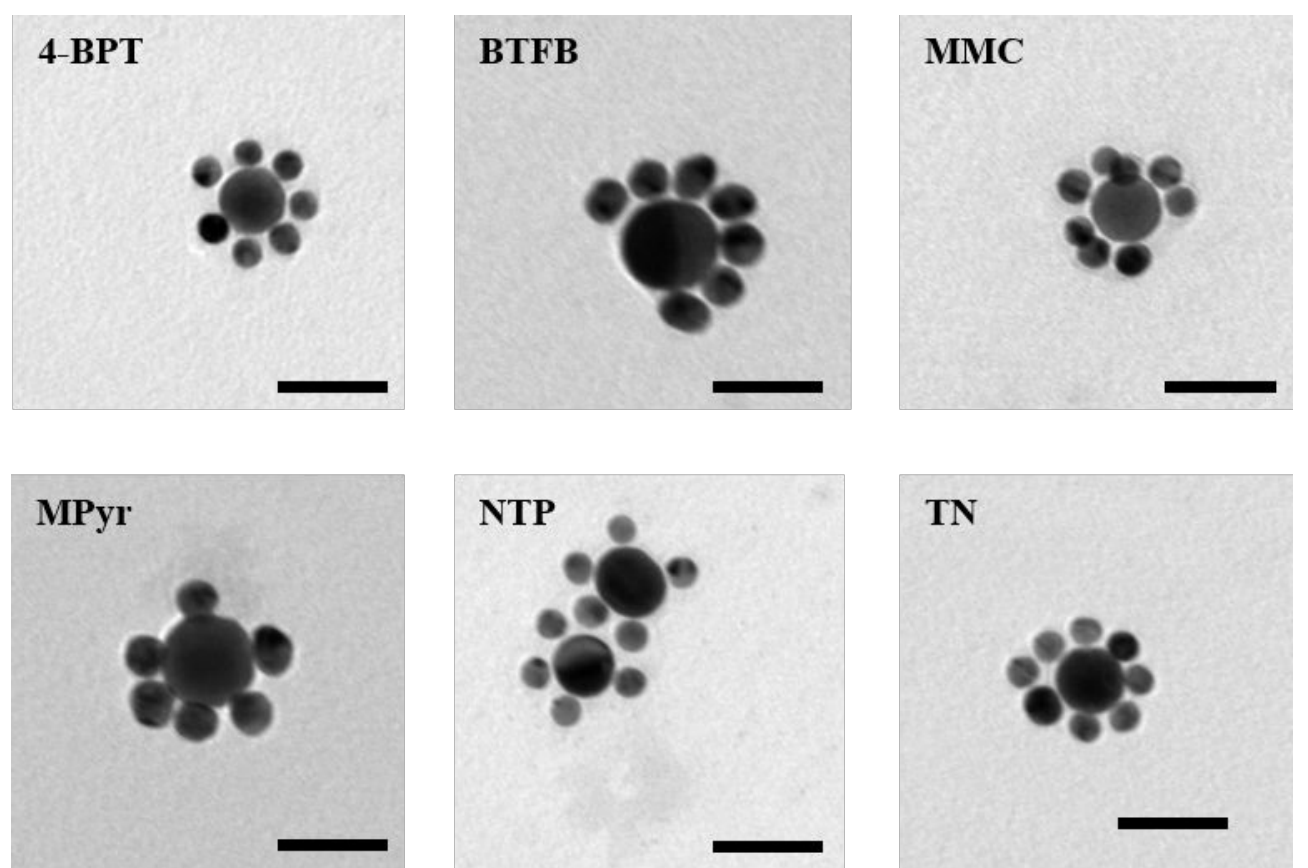

Figure S2. TEM images of $\mathrm{Au} / \mathrm{Au}$ core/satellite nanoparticles with 6 different Raman reporters:

BPT, BTFB, MMC, MPyr, NTP, TN. Scale bar is $50 \mathrm{~nm}$.
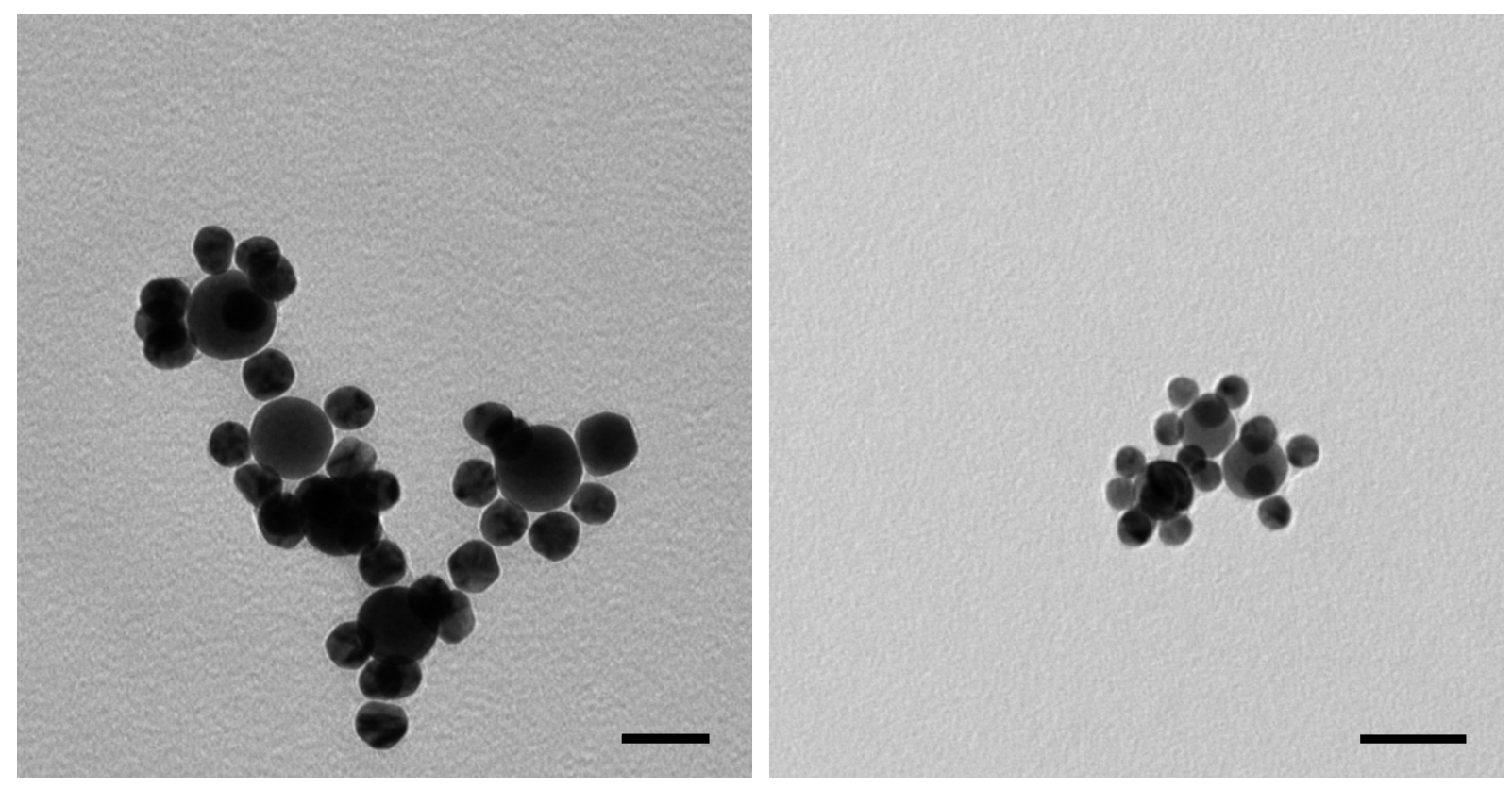

Figure S3. TEM images of $\mathrm{Au} / \mathrm{Au}$ core/satellite nanoparticles with two different core sizes. Left: $42 \mathrm{~nm}$ core/ $17 \mathrm{~nm}$ satellites. Right: $30 \mathrm{~nm}$ core/ $17 \mathrm{~nm}$ satellites. Scale bar is $50 \mathrm{~nm}$. 


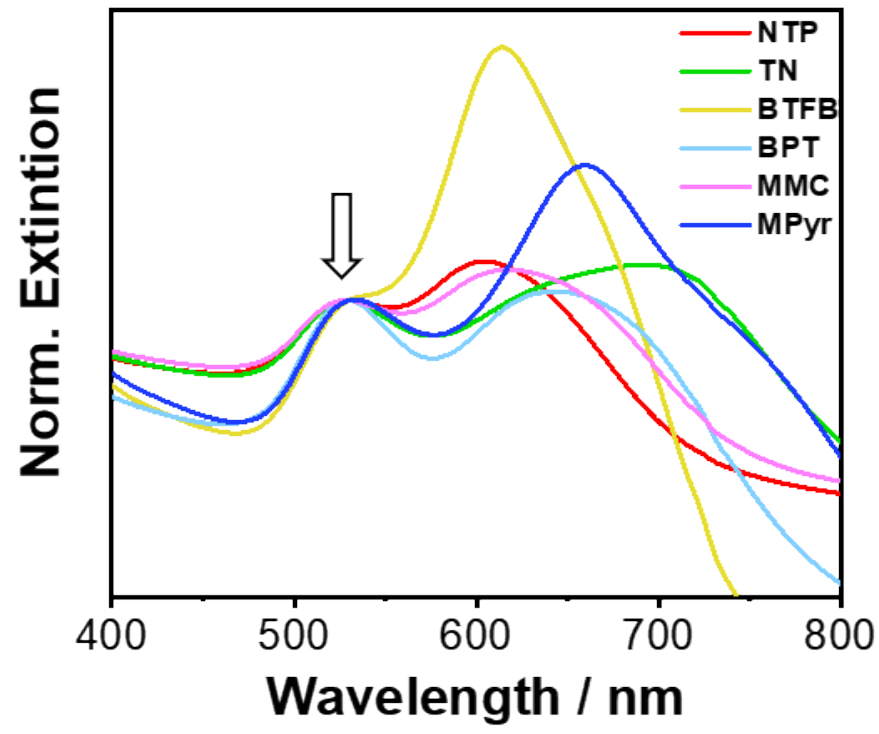

Figure S4. UV/Vis extinction spectra of Au/Au core/satellite nanoparticles with 6 different Raman reporters: BPT, BTFB, MMC, MPyr, NTP, TN, normalized to the extinction at ca. $530 \mathrm{~nm}$ (arrow). 